\title{
A Human-Machine Interface Using Electrical Impedance Tomography for Hand Prosthesis Control
}

\author{
Yu Wu, Student Member, IEEE, Dai Jiang, Member, IEEE, Xiao Liu, Senior Member, IEEE, Richard \\ Bayford, Member, IEEE, and Andreas Demosthenous, Fellow, IEEE
}

\begin{abstract}
This paper presents a human-machine interface that establishes a link between the user and a hand prosthesis. It successfully uses electrical impedance tomography, a conventional bio-impedance imaging technique, using an array of electrodes contained in a wristband on the user's forearm. Using a highperformance analog front-end application specific integrated circuit (ASIC) the user's forearm inner bio-impedance redistribution is accurately assessed. These bio-signatures are strongly related to hand motions and using artificial neural networks, they can be learned so as to recognize the user's intention in real-time for prosthesis operation. In this work, eleven hand motions are designed for prosthesis operation with a gesture switching enabled sub-grouping method. Experiments with five subjects show that the system can achieve $98.5 \%$ accuracy with a grouping of three gestures and an accuracy of $94.4 \%$ with two sets of five gestures. The ASIC comprises a current driver with common-mode reduction capability and a current feedback instrumentation amplifier (that occupy an area of $0.07 \mathrm{~mm}^{2}$ ). The ASIC operates from $\pm 1.65 \mathrm{~V}$ power supplies and has a minimum bio-impedance sensitivity of $12.7 \mathrm{~m} \Omega_{\mathrm{p}-\mathrm{p}}$.
\end{abstract}

Index Terms-Current driver, electrical impedance tomography, human machine interface, hand prosthesis control, instrumentation amplifier.

\section{INTRODUCTION}

$\mathrm{C}$ ONTROL of the hand to perform tasks and to communicate with others is doubtless one of human's most valuable abilities to possess. With the advancement of technology and the ever-increasing fusion of computer and machine into daily life, a seamless human-machine interface (HMI) system that can recognize hand gestures and motions, and allow the control of robotic machines and prostheses to perform dexterous tasks, is a target of research. Once established, this link between humans and machines can greatly enhance the quality of life, with applications ranging from better control of robotics in, for example, surgery operations, restoring a degree of normality to amputees or safely handling hazardous materials.

With such promising motivation, a variety of approaches has been reported to handle hand gesture and motion recognition towards a HMI. The approaches can be classified into two main categories [1]:

1. Image based: It uses a camera to 'see' the gestures and

Manuscript received 22 May, 2018; revised August 09, 2018; and September 25,2018 .

The authors are with the Department of Electronic and Electrical Engineering, University College London, London WC1E 7JE, U.K.

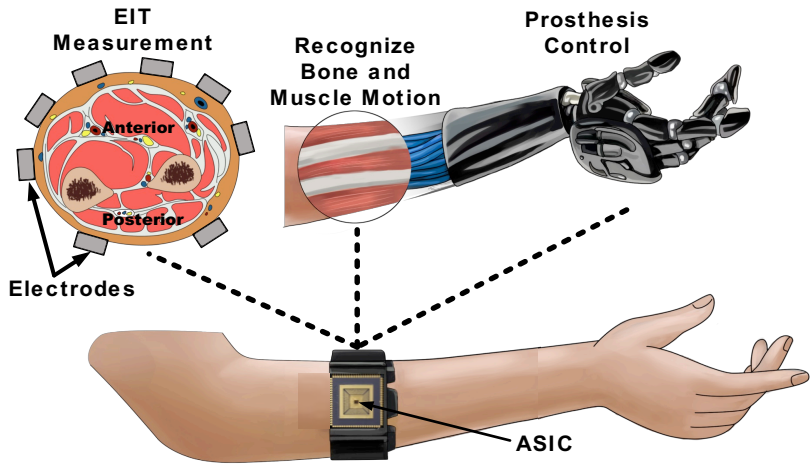

Fig. 1. Proposed EIT based hand prosthesis control system with ASIC.

software to interpret the image. The technology has been developed since the nineties with many reported applications [2]. In addition to cameras, sensors are incorporated into the system to improve sensitivity and accuracy [3]. The limitation of this HMI is the requirement for line-of-sight and may not be suitable for prosthesis-related applications.

2. Non-image based: An alternative approach is to capture the hand motions through a glove equipped with e.g. bend sensors [4]. Glove based HMI has the benefit of being simple and robust but is limited in its range of applications. The most promising HMI method by far is surface electromyography (sEMG) [5]. While hand motion is performed, the muscle cells are neurologically activated and generate voltage potentials. By placing electrodes on the skin surface, the voltage potentials can be measured near these muscle groups. Because different motions activate different muscle groups, successive recorded data can be used for motion classification. This type of HMI can be applied to our daily life as a wearable gadget [6]; it has been dominantly used in active/functional prostheses. According to [5] up to $50 \%$ of upper-limb prostheses are based on sEMG, and this technique can achieve high recognition accuracy [7], [8].

This paper presents a different HMI approach to the ones described above. The proposed system is shown in Fig. 1. As hand motions are strongly and more directly related to the physical movement of muscles and bones in the forearm, the proposed system aims to measure these movements using a bioimpedance imaging technology, namely electrical impedance

(e-mail: yu.wu.09@ucl.ac.uk; d.jiang@ucl.ac.uk; xiao@ucl.ac.uk; r.bayford@ucl.ac.uk; a.demosthenous@ucl.ac.uk). 
tomography (EIT). The electronics of this EIT reader are mounted on a wristband which contains eight electrodes as shown in Fig. 1. The reader features a high-performance, analog front-end application specific integrated circuit (ASIC) to capture the bone and muscle motion through EIT measurements, and uses the data to control a prosthetic hand in real-time.

The rest of the paper is organized as follows. Section II discusses the use of EIT for HMI hand prosthesis control and system design considerations. Section III presents the detailed system design and implementation. Measured results in Section IV demonstrate the successful operation of the EIT-based HMI hand prosthesis system and its medium-term performance. The merits and limits of EIT for HMI hand prosthesis control are discussed in Section V. Concluding remarks are provided in Section VI.

\section{BACKGROUND AND RELATED WORK}

As a promising HMI approach, sEMG has undergone years of development, especially for hand prosthesis [9], [10]. Reported studies cover topics such as: 1) electronic designs for detection of sEMG using discrete components [11] as well as integrated circuit solutions [12], [13]; 2) electrode materials, placement and number of channels [14], [15]; 3) data processing methods for intent interpretation e.g. using support vector machine [16] and neural networks [17]; 4) myoelectric actuator control profiles such as on-off, and proportional schemes [18] as well as grasp force feedback control [19].

Although sEMG has been generally successful, a significant gap remains in making a seamless HMI for hand prosthesis control. The fundamental challenges relate to EMG recording [20]. The EMG amplitude is up to tens of $\mathrm{mV}$ with frequencies up to about $500 \mathrm{~Hz}$. This makes recording susceptible to noise and low frequency interference. Deep muscle activity in the forearm is also difficult to record using sEMG. Alternative ways of recording raw bio-signals that may be merged with sEMG systems are required to enhance system performance.

EIT is a non-invasive, radiation-free bio-impedance mapping technique. It comprises an array of electrodes in a wristband wrapped around the forearm of the subject under test (SUT) as shown in Fig. 1. It can measure and estimate the inner structural conductivity distribution of any enclosed conductive object in the cross-section plane that the band is wrapped around. When hand motion is performed, bones and muscles move under the skin resulting in a temporal alteration of conductivity distribution. This causes inner-impedance variations that can be recorded and ultimately related back to the hand motions.

To better understand EIT operation, an eight electrode EIT approximate resistive mesh model based on [21] is shown in Fig. 2(a). It operates by injecting a constant sinewave current via a pair of electrodes and recording the voltages on the SUT's surface with the remaining electrodes. The amplitude and phase of these induced voltages vary according to the internal conductivity re-distribution, e.g. muscle movement.

When designing a high performance EIT system the following specifications must be considered:

1. As the SUT is inhomogeneous, a differential current
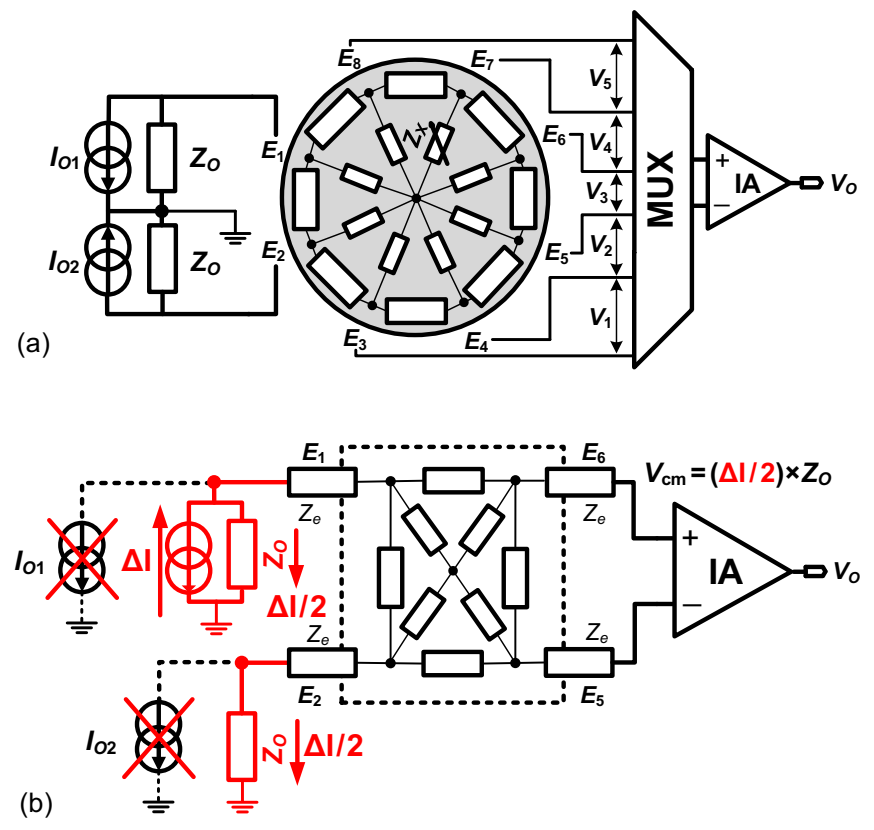

Fig.2. EIT models: (a) An approximate EIT resistive mesh model; (b) Approximate tetra-polar bio-impedance measurement model with imbalanced current source. $E_{i}$ are electrodes, $Z_{e}$ are the skin contact resistances and $V_{\mathrm{cm}}$ is the common mode signal at the inputs of the IA.

driver [22] should be used as shown at electrodes $E_{1}$ and $E_{2}$ in Fig. 2(a). This reduces the common mode signal on the measuring side and enables the instrumentation amplifier (IA) to detect the differential signal between electrodes e.g. differential voltage $V_{3}$ as shown.

2. The IA should have good common mode rejection ratio (CMRR) as EIT measures the dynamic changes between differential signals e.g. variation in voltage $V_{5}$ when impedance $Z_{X}$ changes.

3. Bio-impedance should be measured through a tetra-polar scheme (i.e. not involving the contact impedance from the current driver during voltage measurements).

4. The differential current driver should be tightly matched as any imbalance between the source and sink currents produces a residual current $\Delta \mathrm{I}$ that can flow through the high impedance node $Z_{O}$ to ground producing unwanted common mode signals [22], [23] as shown in Fig. 2(b).

5. For accurate information, the measured data should be I$\mathrm{Q}$ demodulated to provide the real and imaginary parts of the bio-impedance.

Originally developed as an imaging technique, EIT has recently been used for HMI related applications [24], [25]. Our first prototype reported in [26] overcame some of the design challenges listed above with hardware improvements over the AD5933 (a commercial bio-impedance analyzer chip using a two-electrode measurement scheme) based systems in [24], [25]. However, the overall system was cumbersome and power demanding. While low power solutions are available for most of the components such as digital-to-analog converter (DAC), the bottleneck is the analog front-end where the selected offthe-shelf integrated circuits for the high CMRR IA and the fully differential current driver require at least $\pm 5 \mathrm{~V}$ to operate with no obvious low-voltage substitutions. Especially for the current driver, the design choices and circuit performance at discretelevel implementation are very limited [27]. To this end, an 


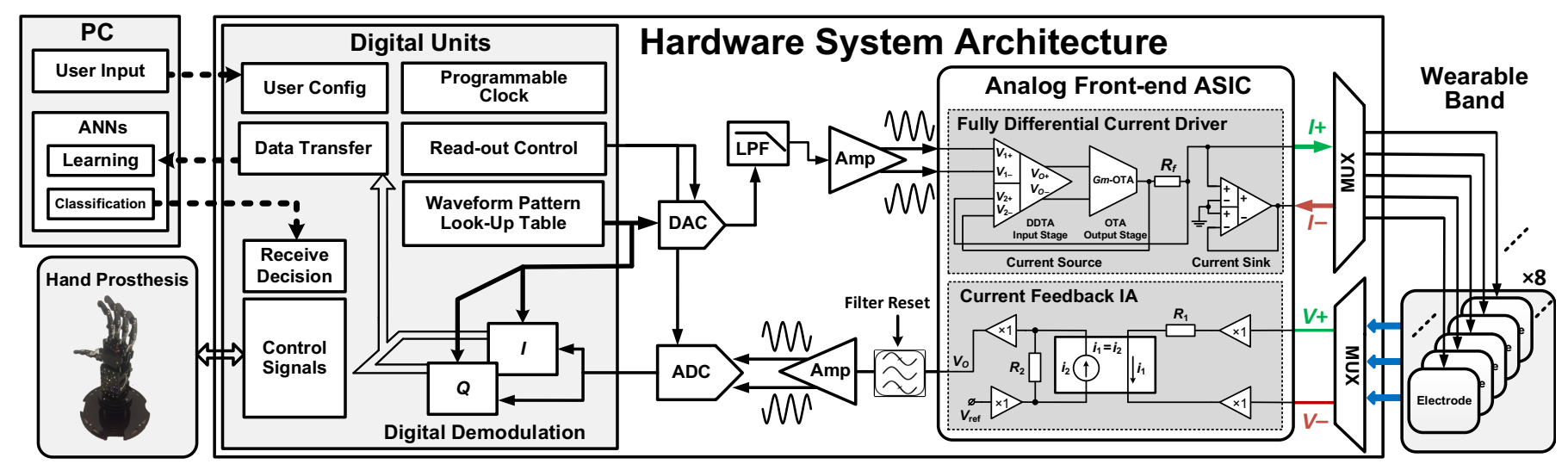

Fig. 3. Overall HMI system architecture.

analog front-end ASIC has been designed towards developing a fully integrated HMI system for hand prosthesis control.

\section{System Design AND IMPLEMENTATION}

\section{A. System Architecture}

The overall system architecture is shown in Fig. 3. It comprises three parts: i) hardware EIT system, ii) software on the $\mathrm{PC}$ for data processing and pattern recognition, and iii) hand prosthesis control unit. The EIT reader is attached to the wearable wristband and can operate the eight electrodes inside the band.

The DAC generates a voltage signal, which is filtered and sent to the fully differential current driver in the ASIC. The current driver block diagram is shown in Fig. 3 and has current source and current sink parts. The differential difference transconductance amplifier (DDTA) input stage of the current source part regulates the source current $I+$ by measuring the input signal and the voltage across resistor $R_{f}$ and produces a voltage to the operational transconductance amplifier (OTA) output stage according to the measured differences [28]. To drive the load differentially, a current $I-$ is provided by the current sink part. It operates by measuring the common mode voltage across the load and through feedback, this part of the circuit adjusts its output until the common mode voltage is reduced to zero.

The EIT voltages are measured through the current feedback IA in the ASIC. The basic functional diagram of the IA is shown in Fig. 3. The input voltage is applied via buffers to resistor $R_{1}$. The resulting current through $R_{1}$ is transferred to resistor $R_{2}$. The output voltage $V_{O}$ is the sum of voltage $V_{\text {ref }}$ and the voltage across $R_{2}$ provided via buffers. This architecture avoids the need multiple matching resistors that are used in conventional designs. Following band-pass filtering the output is digitized for I-Q demodulation.

After receiving the user input through the UART link, EIT measurements are initiated. On completion, demodulated data carrying hand motion information is transferred back to the PC for pattern learning and then classification. When given a classification, it is passed on to the microcontroller on the prosthesis hand. The control unit sends out pulse width modulation (PWM) signals to ultimately recreate the same hand grip pattern that the user is performing when EIT measurements are taken.

\section{B. Analog Front-end ASIC}

The current driver transistor level schematic is shown in Fig. 4(a). For the current source circuit, the input voltages are measured using transistors M1 and M2 in the DDTA input stage, while the voltage across $R_{f}$ is measured through M3 and M4. The drain currents of M1, M3 and M2, M4 are summed together and mirrored through M5 and M6 to the two output branches to provide the differential output:

$$
V_{O+}-V_{O-}=A_{\mathrm{ol}} \cdot\left\{\left(V_{1+}-V_{1-}\right)-\left(V_{2+}-V_{2-}\right)\right\}
$$

where $A_{\mathrm{ol}}$ is the open loop gain of the DDTA input stage that is enhanced by the cascoded output branch. The differential output is connected to the OTA output stage whose output is connected to $R_{f}$ to provide linear feedback. The source current $I+$ can be written as:

$$
I+=\left(V_{1+}-V_{1-}\right) \cdot \frac{A_{\mathrm{ol}} \cdot G m_{\mathrm{ota}}}{1+A_{\mathrm{ol}} \cdot G m_{\mathrm{ota}} \cdot R_{f}}
$$

where $G m_{\text {ota }}$ is the transconductance of the OTA output stage and $V_{1+}-V_{1-}$ is the input voltage of the current source circuit.

The current sink circuit, a two-stage differential difference amplifier, is shown in Fig. 4(a). The gates of M17 and M19 are connected across the load $Z_{L}$, and their drain currents are summed and mirrored to the M22-M20 branch; the gate terminals of M18 and M20 are grounded. With the amplifier's output also connected to the gate of M19, this feedback regulates the amplifier's output to generate a voltage that would sink a current $I-$ equal to $-(I+)$ and provide a fully differential voltage across the load [29].

The transistor level schematic of the IA based on [30] is shown in Fig. 4(b). It is realized by two resistive-degenerated transconductors. For the input transconductor, transistor M6M8 and MC, MD form a current feedback loop that forces the drain currents of M1 and M2 to be equal through the current mirrors MA-MD. As a result, the input stage acts as a unity gain buffer and the differential input voltage is applied across $R_{1}$. For the output transconductor, transistors M9-M21 form another current feedback loop. This feedback also forces the drain currents of M9, M10 to be equal. Since the differential current in $\mathrm{ME}, \mathrm{MF}$ is mirrored from MA, MB, the current in $R_{2}$ is identical to the current in $R_{1}$ and the output voltage $V_{O}$ appears across $R_{2}$, resulting in a voltage gain of $R_{1} / R_{2}$. Voltage $V_{\text {ref }}$ is used to set the reference level of the output. 


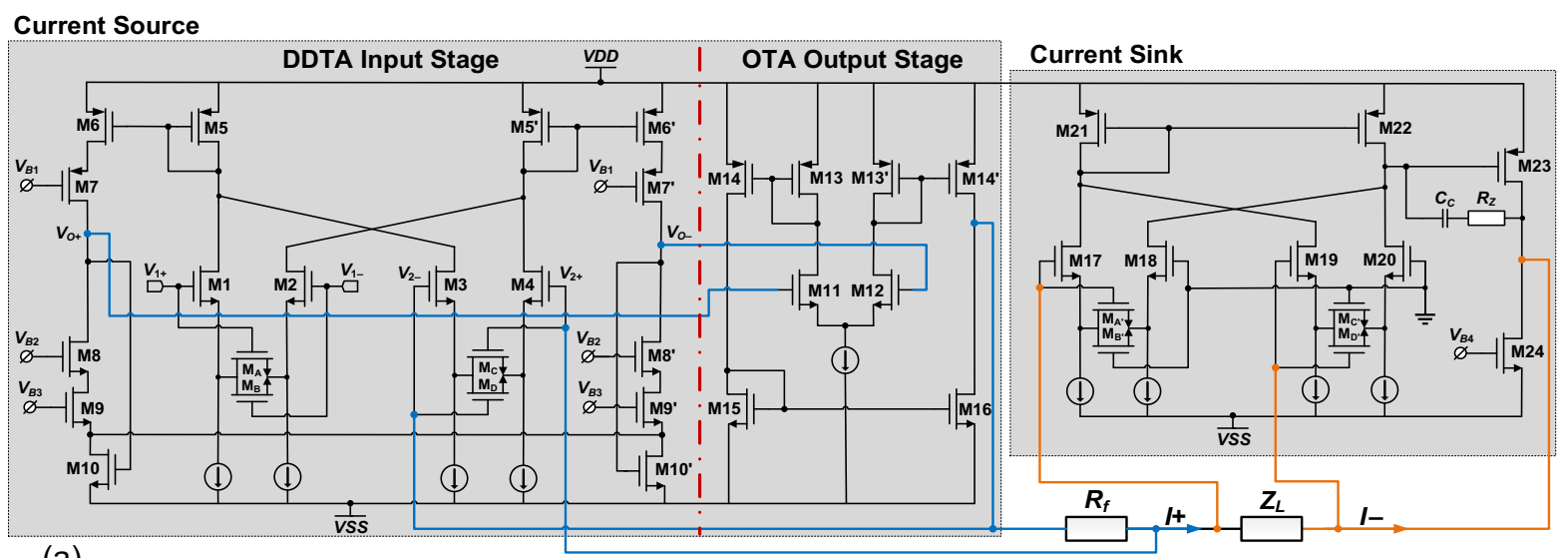

(a)

(b)

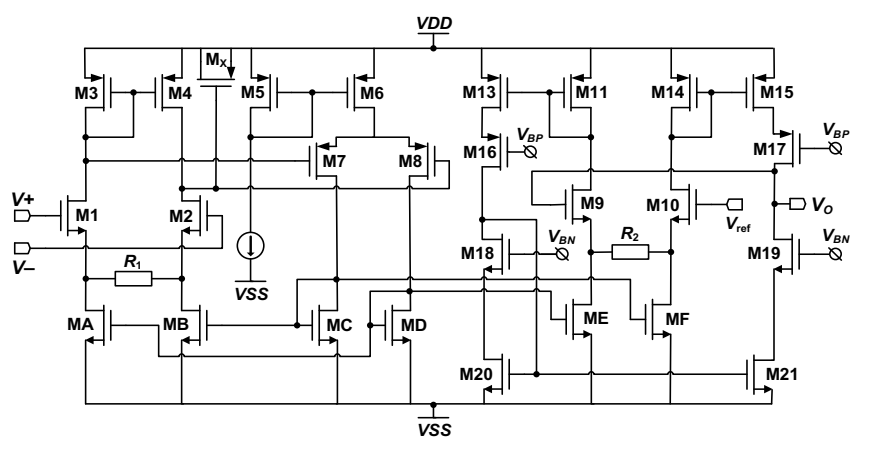

Fig. 4. Transistor level schematic of the current driver (a) and IA (b).

The input-referred voltage noise of the IA is defined by:

$$
\frac{v_{i N}^{2}}{\Delta f}=4 k T R_{1}+2 \cdot 4 k T \cdot \frac{2}{3}\left(\frac{1}{g_{m 1,2}}+\frac{g_{m 3,4}}{g_{m 1,2}^{2}}\right)
$$

where $g_{m i}$ is the small-signal transconductance of transistor $i$, $k$ is Boltzmann's constant, $T$ is the absolute temperature, and the noise is measured over a bandwidth of $\Delta f$. From (3) it is observed that the noise can be minimised by having a small $R_{1}$. $\mathrm{R} 1$ is also related to the differential input range, defined as 2 . $R_{1} \cdot I_{d 1,2}$. There is a design trade-off between input range, noise performance and power consumption. Based on resistive mesh simulation and comparing the specification of the IA in [26], the input range was designed to be $100 \mathrm{mV}_{\mathrm{p}-\mathrm{p}}$; the biasing current was set to $25 \mu \mathrm{A}, R_{1}=2 \mathrm{k} \Omega$ and $R_{2}=20 \mathrm{k} \Omega$. With the gain of the IA set to $10 \mathrm{~V} / \mathrm{V}$, its simulated input-referred noise was $10 \mu \mathrm{V}_{\text {rms }}$ over the bandwidth $25 \mathrm{kHz}$ to $1 \mathrm{MHz}$.

Capacitive mismatch is the primary reason for a limited CMRR in IA design. To enable a high CMRR at high frequencies, apart from random mismatches which could be reduced by a symmetrical layout, a simple but effective method is to insert a neutralization capacitor, implemented by transistor M4' as shown in Fig. 4(b), to equalize the capacitances at the drains of M3 and M4. The ratio of the width of Mx to the width of the current mirror transistors (M3, M4) is set to approximately 4/3 [30].

\section{Digital Control and IQ Demodulation}

The ASIC is connected to the digital circuits through a pair of data converters and the analog back-end comprising standard analog filters and two single-to-differential amplifiers as shown in Fig. 3, using off-the-shelf components. The digital circuits

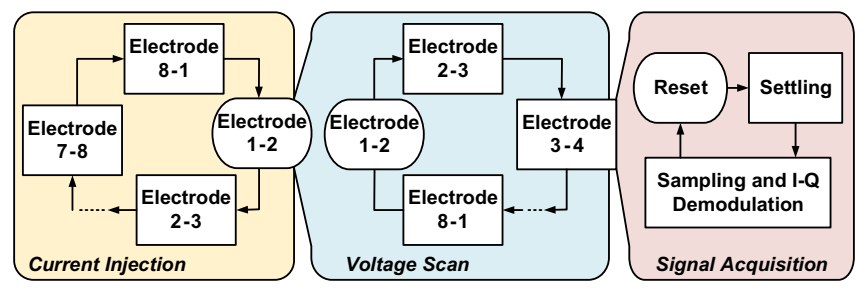

Fig. 5. State machine for controlling the EIT operation.

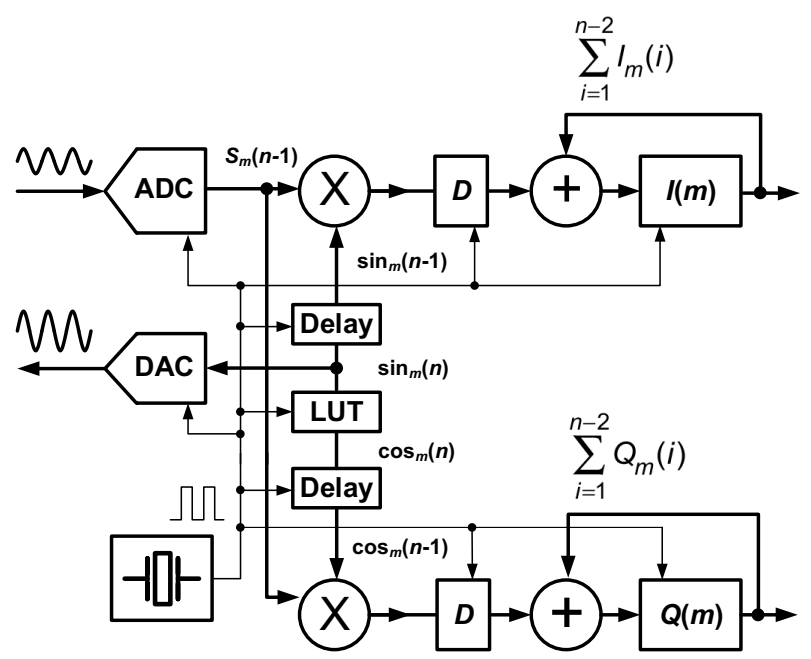

Fig. 6. State machine for controlling the EIT operation.

control the EIT operation with a state machine comprising three nested loops, as shown in Fig. 5, managing the electrode scan for current drive, voltage readout, and signal acquisition and 


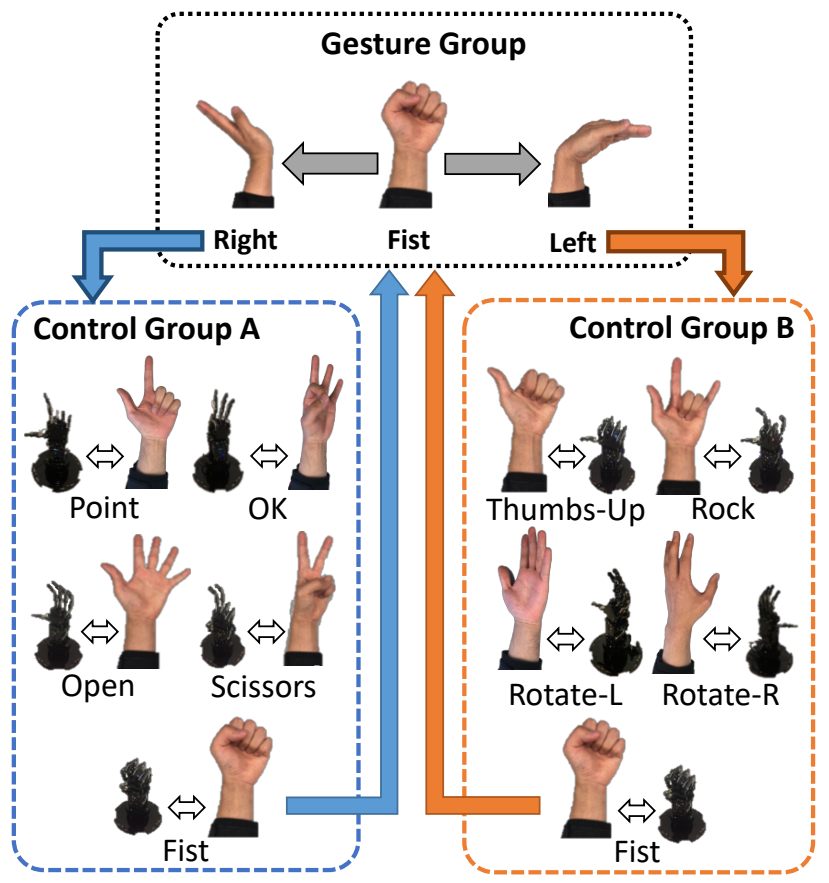

Fig. 7. Hand prosthesis control with two sub-control groups with gesture enabled switching method.

processing. The outer loop controls the current drive, which has eight states, each selecting a pair of adjacent electrodes to be multiplexed to the current driver. Each state in the outermost loop triggers the middle loop for voltage scan. The middle loop runs a full cycle before the outer loop moves to the next state. The outer loop completes a full cycle to generate one complete dataset. The middle loop also has eight states controlling the multiplexing of eight pairs of adjacent electrodes to the IA. Each state in the middle loop triggers the inner loop for signal acquisition. There are three states in the inner loop. After the middle loop has switched the IA inputs to a new pair of electrodes, the inner loop first resets the band-pass filter in the analog back-end to the reference voltage in order to avoid large signal fluctuation that might require a long settling time. After the reset, the inner loop allows the voltage at the input of the $\mathrm{ADC}$ to settle before starting sampling and I-Q demodulation. Once the inner loop completes the sampling and I-Q demodulation state, the middle loop moves to the next state, which triggers a new cycle in the inner loop. A full cycle of the inner loop takes $1.4 \mathrm{~ms}$, hence generating one dataset takes $89.6 \mathrm{~ms}$.

Fig. 6 shows the implementation of the sampling and I-Q demodulation unit. The I-Q demodulation, using a digital lockin amplifier [31], synchronizes to the DDS-based signal generator that drives the current driver. For example, for a specific state in the middle loop, $m$, the digital logic reads out samples from a lookup table (LUT) of a pre-stored sinewave, $\sin _{m}(n)$, and its $90^{\circ}$ phase shift, $\cos _{m}(n)$. The $\sin _{m}(n)$ samples are converted by the DAC into a sinewave for the current driver. For every LUT readout, the digital lock-in amplifier receives a sample from the ADC, $S_{m}(n-1)$, taking into account the delay from the data converters and the analog front-end. $S_{m}(n-1)$ multiplies in parallel with the previous readout samples from the LUT, $\sin _{m}(n-1)$ and $\cos _{m}(n-1)$. The results from the
Neural Networks Training

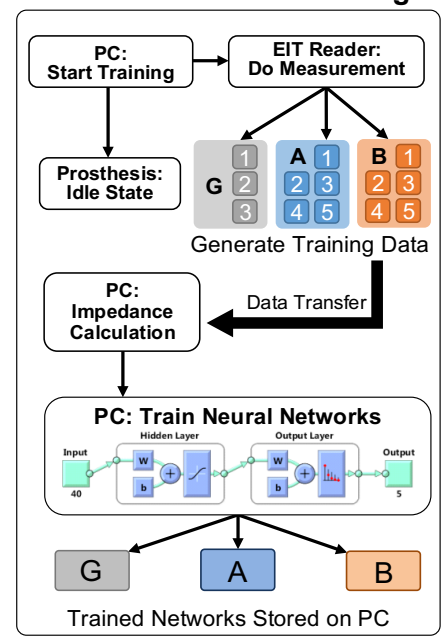

Prosthesis Operation

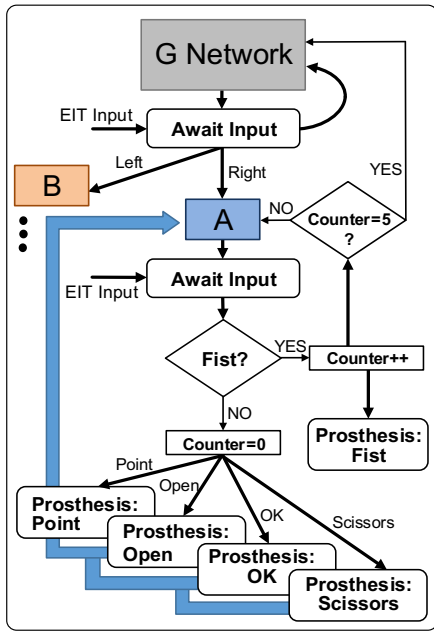

Fig. 8. System control flow chart for training and prosthesis operation.

multipliers accumulate with all the previous results from the same electrode position, $m$, to derive the real and imaginary vectors $I(m)$ and $Q(m)$. For each $m$, in total 128 samples are taken for deriving $I(m)$ and $Q(m)$ at a sampling rate of $3 \mathrm{MS} / \mathrm{s}$.

\section{Hand Prosthesis Motion Control}

A servo-motor based hand prosthesis is used in this study. It has six servo-motors of which five are inside the prosthesis arm and are responsible for finger movement and one underneath the holding station for rotating the whole prosthesis hand. An Arduino-Nano microcontroller is placed on the station for prosthesis motion control. The previous study [26] showed that groups of five hand gestures give optimal performance, and it also suggested a round robin sub-grouping method to increase the total number of gestures recognizable but did not provide an intuitive way for robin rotation.

In this work, nine hand gestures divided into two sub-control groups are proposed that can be recreated by the hand prosthesis, and two other hand gestures (with no corresponding prosthesis motion) to implement the group selection without additional hardware. The control scheme is shown in Fig. 7. The user can perform 'Left' or 'Right' gesture to select the desired sub-control group. Each sub-control group has five gestures that the user can perform and 'Fist' is a shared gesture in all groups, to allow sub-group re-selection.

Due to individual differences, each user is required to train specific neural networks before their hand motion is recognizable by the system. Fig. 8 shows the complete system control flow chart. It is divided into two main phases: the data training phase and the prosthesis operation phase. The system uses neural networks to recognize the bio-impedance patterns measured by the EIT reader. The neural networks are provided by MATLAB's neural network toolbox-pattern recognition tool. During the training, the prosthesis hand is in the idle state. The user needs to hold the hand gesture while the EIT reader continuously carries out measurements until sufficient data is recorded. The system records three sets of data for the 'Gesture' group and five sets of data for each sub-group A and B. Each time the EIT reader takes a measurement, it sends all demodulated I-Q data to the PC where the data is converted to 


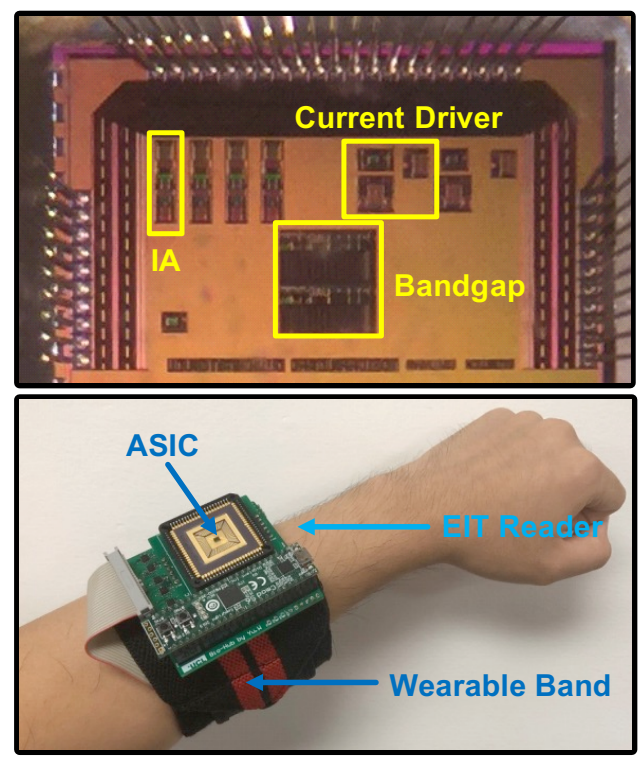

Fig. 9. ASIC micrograph with individual blocks labeled and the complete EIT reader with the ASIC mounted on a daughter board and a FPGA stacked on top of the motherboard.

TABLE I.

ASIC MEASURED PERFORMACE

\begin{tabular}{|c|c|c|}
\hline Parameter & Current Driver & IA \\
\hline Gm/Gain & $3.97 \mathrm{~mA} / \mathrm{V}$ & $10 \mathrm{~V} / \mathrm{V}$ \\
\hline Bandwidth & $500 \mathrm{kHz}$ & $200 \mathrm{kHz}$ \\
\hline Output Current & $\mathrm{Up}$ to $1 \mathrm{~mA}_{\mathrm{p}-\mathrm{p}}$ & - \\
\hline $\begin{array}{l}\text { Output } \\
\text { Impedance }\end{array}$ & $\begin{array}{c}750 \mathrm{k} \Omega \\
\text { (at } 500 \mathrm{kHz} \text { ) }\end{array}$ & - \\
\hline $\begin{array}{l}\text { Common Mode } \\
\text { Reduction / CMRR }\end{array}$ & $\begin{array}{c}97.5 \% \\
{[29]}\end{array}$ & $\begin{array}{c}80 \mathrm{~dB} \\
\text { (at } 200 \mathrm{kHz} \text { ) }\end{array}$ \\
\hline $\begin{array}{l}\text { Differential Input } \\
\text { Signal Range }\end{array}$ & - & $100 \mathrm{mV}_{\mathrm{p}-\mathrm{p}}$ \\
\hline THD & $42 \mathrm{~dB}$ & $53 \mathrm{~dB}$ \\
\hline $\begin{array}{c}\text { Input-Referred Noise } \\
(25 \mathrm{kHz}-1 \mathrm{MHz})\end{array}$ & - & $9 \mu \mathrm{V}_{\mathrm{rms}}$ \\
\hline
\end{tabular}

forty bio-impedance values (in decimal) that are labeled and stored for training. Once the stored data is learnt, functions that represent the trained neural networks can be generated and used for classification during the prosthesis operation phase. There are three trained neural networks as shown: $\mathrm{G}$ for 'Gesture' group and A and B for the two sub-control groups. Only one neural network is online at a given time.

In the prosthesis operation phase, the EIT reader continuously takes measurements every $200 \mathrm{~ms}$ until the user terminates the operation. Measured data is passed to the trained neural network for classification. The system starts with $G$ network as shown in Fig. 8 and progresses to control group A or B if 'Left' or 'Right' gesture is recognized. The software gives indications as to which group is online and pauses for $3 \mathrm{~s}$ when entering a sub-control group, so that the user can regesture according to the selected groups. As the flow chart is identical for groups A and B, only A is shown in Fig. 8. The system employs a counter return method so that when five consecutive 'Fist' gestures are detected, the online trained

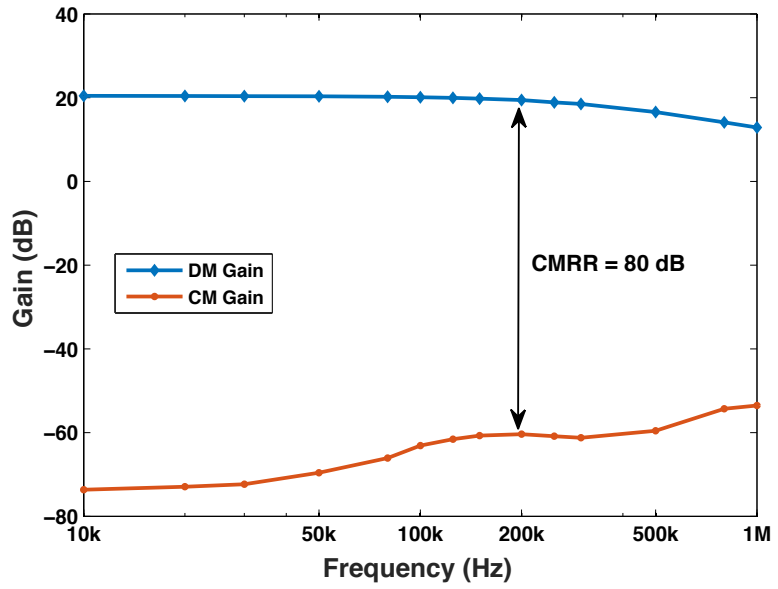

Fig. 10. Measured common mode and differential mode gains of the IA.

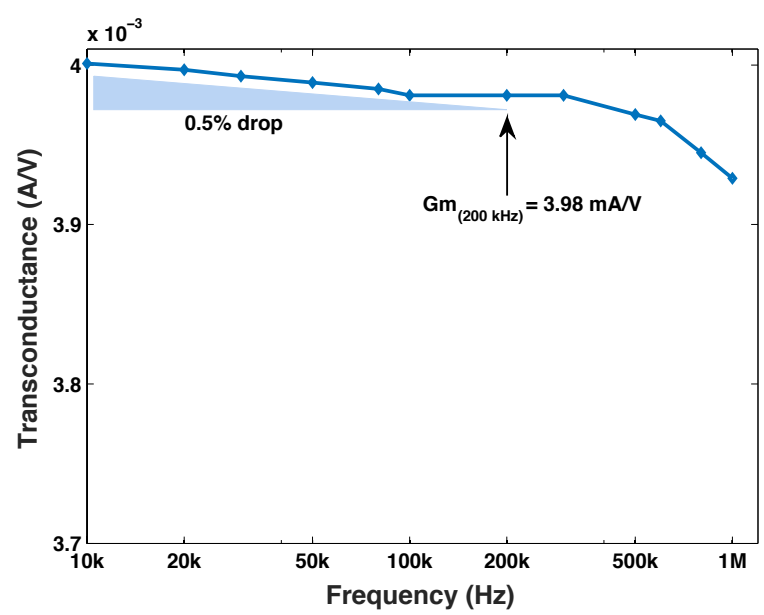

Fig. 11. Measured transconductance and bandwidth of the current driver with a $1 \mathrm{k} \Omega$ load.

neural network returns to $G$ with the prosthesis set in the 'Fist' gesture. Until such condition occurs, the system stays in the sub-control group. When another gesture is classified, the counter resets and the microcontroller operates the hand prosthesis according to the classification output.

\section{Measured Results}

\section{A. Analog Front-End ASIC}

The ASIC is designed in $0.18-\mu \mathrm{m}$ CMOS technology and operates from $\pm 1.65 \mathrm{~V}$ power supplies. The ASIC micrograph is shown in Fig. 9(a) (the current driver and IA occupy a die area of $0.07 \mathrm{~mm}^{2}$ ) and Fig. 9(b) the complete EIT reader system (which includes an FPGA development board). The ASIC performance is summarized in Table I. The system consumes $340 \mathrm{~mW}$ of which $14 \mathrm{~mW}$ is in the ASIC. A fully integrated design [32] would allow the power consumption to be substantially reduced.

The IA measured maximum differential input signal range is $100 \mathrm{mV}_{\mathrm{p}-\mathrm{p}}$, and at $200 \mathrm{kHz}$ the output has a total harmonic distortion (THD) of $53 \mathrm{~dB}$. The measured input-referred voltage noise is $9 \mu \mathrm{V}_{\mathrm{rms}}$ from $25 \mathrm{kHz}$ to $1 \mathrm{MHz}$. As shown in 


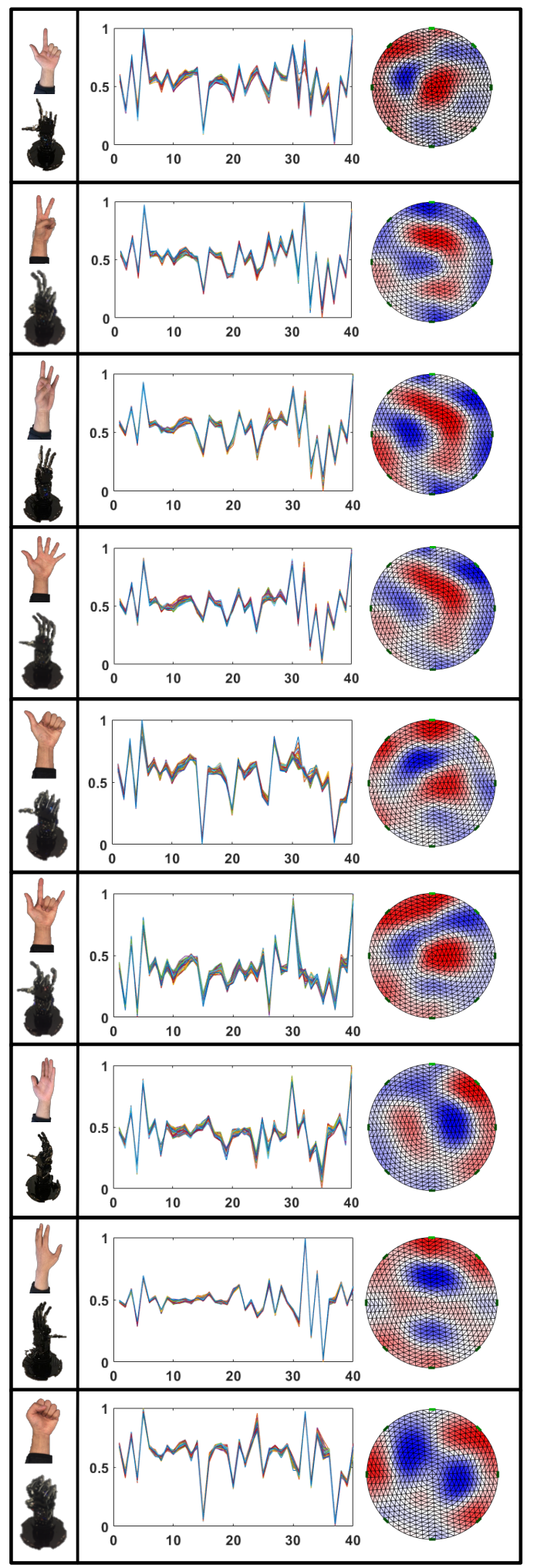

Fig. 12. All gestures with corresponding prosthesis motion, and 50 datasets of 40 measured impedance combinations plotted with corresponding EIT images.

Fig. 10 the IA has a gain of $20 \mathrm{~dB}$ up to $200 \mathrm{kHz}$ and a common mode gain of $-60 \mathrm{~dB}$ (input common mode signal of $1 \mathrm{~V}_{\mathrm{p}-\mathrm{p}}$ ), providing $80 \mathrm{~dB}$ CMRR at $200 \mathrm{kHz}$. The transconductance (and bandwidth) of the current driver was measured by outputting a $1 \mathrm{~mA}_{\mathrm{p}-\mathrm{p}}$ current into a $1 \mathrm{k} \Omega$ load; the results are shown in Fig. 11. The transconductance is maintained at $3.98 \mathrm{~mA} / \mathrm{V}$ at
$200 \mathrm{kHz}$ and with only a $0.5 \%$ drop from the initial value at $10 \mathrm{kHz}$. The measured output impedance of the current driver is $750 \mathrm{k} \Omega$ at $500 \mathrm{kHz}$. The current driver is capable of reducing $97.5 \%$ of the common mode signal when compared with current drivers without common mode feedback [29]. The ASIC has a minimum bio-impedance measurement sensitivity of $12.7 \mathrm{~m} \Omega_{\mathrm{p} \text { - }}$ $\mathrm{p}$ (ratio of IA noise level to maximum output current) and can be used to develop a high performance EIT system with a bandwidth up to $200 \mathrm{kHz}$.

\section{B. System Testing and Results}

Five volunteers were recruited, two females and all right handed, for system testing. The wristband was worn on the left forearm and after applying a standard medical grade conductive ECG gel the training could begin (the gel moisturized the skin for better electrical contact).

System testing started with gathering data for neural network training as detailed in Section III-D. Each volunteer was asked to perform the eleven gestures shown in Fig. 7 with 150 datasets taken for each gesture. The collected data was then grouped as shown in Fig. 7 for neural network training. After training was completed, the volunteer was asked to first perform all gestures, starting from group $\mathrm{G}$ and entering group $\mathrm{A}$ and performing all gestures in the group. Afterward, the volunteer could exit the control group and then re-enter group B to repeat the same procedure. Lastly, the volunteer returned to the 'gesture' group. Once this full cycle was completed, the volunteer could perform the gestures at will and the same hand grip pattern could be recreated by the hand prosthesis.

To illustrate how EIT could differentiate between gestures intuitively, a set of measured bio-impedance values were plotted and visually inspected together with a reconstructed EIT image. Shown in Fig. 12 is the data from the first volunteer as an example. Forty measured bio-impedance values in 50 datasets were normalized and plotted with an EIT image reconstructed using the average of these datasets. By visual inspection, it can be seen that each gesture has its own distinctive patterns reflected on both the bio-impedance plots as well as the EIT images.

The system accuracy was evaluated with the cross-validation method using the 150 datasets previously captured. The first 50 datasets were used to train the neural network. Then an additional test was performed using the remaining 100 datasets. Each volunteer's data produced three confusion matrixes and by averaging the results, three overall confusion matrixes could be obtained as shown in Fig. 13. Group $G$ achieved an accuracy of $98.5 \%$ (it is necessary that this group can provide the highest accuracy for sub-control group switching). Group A achieved an overall accuracy of $92 \%$, where confusion occurred between 'Point' and 'Scissors' and between 'OK' and 'Open'. Group B achieved an overall accuracy of $97 \%$ with little confusion occurring between 'Fist' and 'Thumbs-Up'. Overall, these two groups have an average accuracy of $94.4 \%$, and with the result of group $\mathrm{G}$, the overall system accuracy is $95.8 \%$.

Table II provides a comparison between HMI systems, both using sEMG and EIT, for hand gesture recognition and prosthesis control. For sEMG, the system that uses a similar number of acquisition channels (sEMG requires two electrodes per channel) was selected together with hand gesture pools for comparison. As shown, the HMI system reported in this work 
Group A

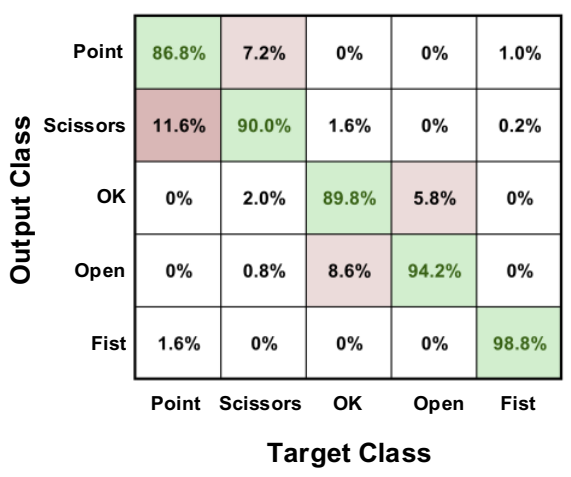

Group B

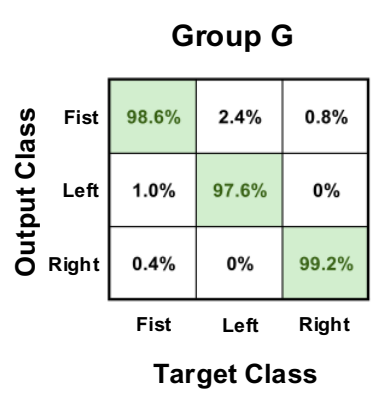

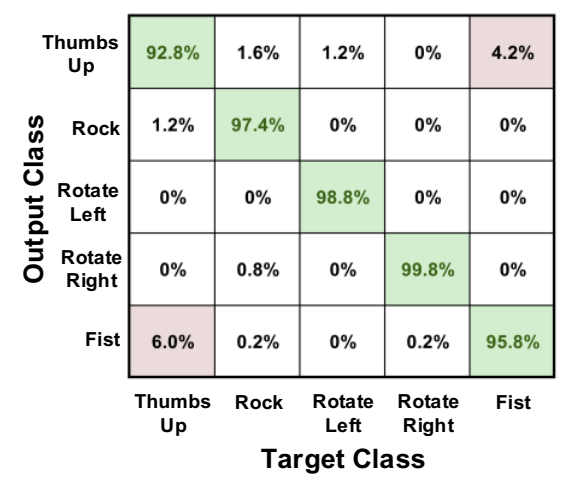

Fig. 13. Confusion matrixes for the three gesture groups.

TABLE II.

COMPARISON WITH OTHER WORK

\begin{tabular}{|c|c|c|c|c|c|c|}
\hline Parameter & {$[\mathbf{9 ]}$} & {$[\mathbf{1 0}]$} & {$[\mathbf{1 3}]$} & {$[\mathbf{2 4}]$} & [25] & This Work \\
\hline Channels $^{1}$ & 6 & 8 & 8 & 8 & $8-32$ & 8 \\
\hline Technique & sEMG & sEMG & sEMG & EIT & EIT & EIT \\
\hline Hand Gestures & 8 & 7 & 7 & $8+5^{2}$ & 11 & $5+5+3$ \\
\hline Classifier & LDA $^{3}$ & KNN & SVM & SVM & SVM & ANN \\
\hline Real-Time & No & Yes & Yes & Yes & Yes & Yes \\
\hline Accuracy & $98 \%$ & $89 \%$ & $90 \%$ & $87 \%$ & $88.5-94.3 \%$ & $95.8 \%$ \\
\hline System Bandwidth & $500 \mathrm{~Hz}$ & $1 \mathrm{kHz}$ & $1 \mathrm{kHz}$ & $100 \mathrm{kHz}$ & $100 \mathrm{kHz}$ & $200 \mathrm{kHz}$ \\
\hline
\end{tabular}

${ }^{1}$ Two EMG electrodes required for one channel recording.

${ }^{2}$ Gesture multi-groups used.

${ }^{3}$ Linear discriminant analysis.

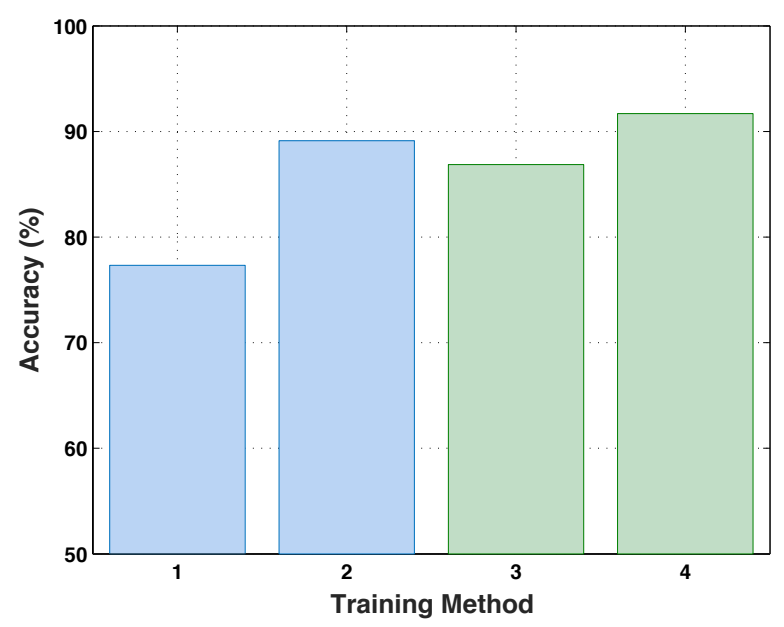

Fig. 14. Single-user multi-session accuracy test results.

has higher accuracy and, benefiting from the ASIC, it can achieve a higher bandwidth, and potentially be further integrated into a system-on-chip solution.

\section{Preliminary Medium-Term Performance}

It was observed that for a single user even with the same predefined gesture, a gesture could be performed with differences (e.g. stretch open and relaxed open). As the system measures the bio-impedance alterations, such differences can reduce system accuracy. This behavior tends to worsen over time as

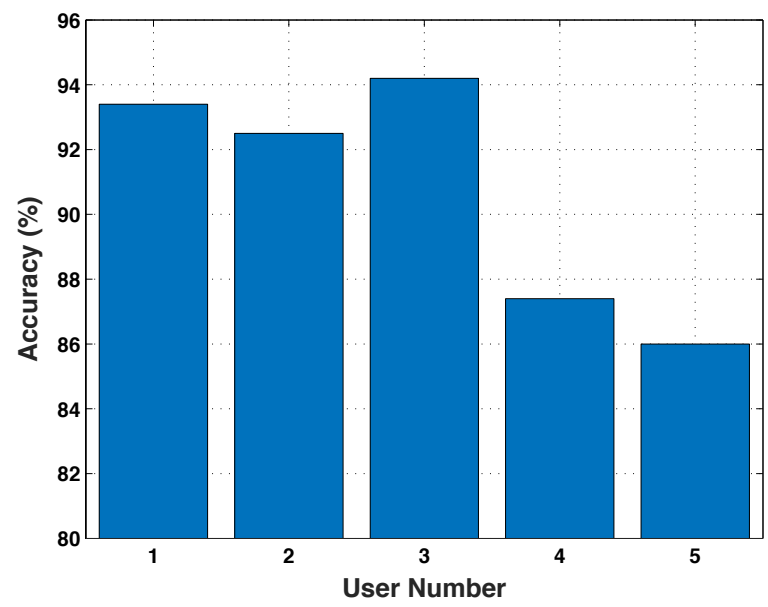

Fig. 15. Multi-user accuracy reproducibility test results.

the user is more likely, but unintentionally, to perform the same gesture with differences. To maintain system accuracy, either data from multiple sessions could be used at the start of training or retraining of the neural network over time.

The reliability of the system accuracy was evaluated over multiple sessions. Fifty sessions were carried out by a volunteer; 50 datasets were taken for each gesture and 5 gestures were taken in one session using group A gestures which had the lowest accuracy in the tests in Section IV-B. Between sessions, there was a two minute break. Using these datasets from fifty 
TABLE IV.

COMPARISON OF MACHINE LEARNING ALGORITHMS

\begin{tabular}{|c|c|c|c|c|c|c|}
\hline & \multicolumn{6}{|c|}{ Support Vector Machine (SVM) } \\
\hline Method & Linear SVM & Quadratic SVM & Cubic SVM & Fine Gaussian & Medium Gaussian & Coarse Gaussian \\
\hline Accuracy & $87.3 \%$ & $91.2 \%$ & $91.4 \%$ & $89.1 \%$ & $89.8 \%$ & $72.7 \%$ \\
\hline Training Time & \multicolumn{6}{|c|}{ K-Nearest Neighbors (KNN) } \\
\hline Method & Fine KNN & Medium KNN & Coarse KNN & Cosine KNN & Cubic KNN & Weighted KNN \\
\hline Method & \multicolumn{3}{|c|}{ Artificial Neural Network (ANN) - 10 Hidden Neurons } & \multicolumn{3}{|c|}{ ANN - 100 Hidden Neurons } \\
\hline Accuracy & \multicolumn{3}{|c|}{$88.8 \%$} & \multicolumn{3}{|c|}{$91.1 \%$} \\
\hline Training Time & \multicolumn{3}{|c|}{$1.0 \mathrm{~s}$} & \multicolumn{3}{|c|}{$4.0 \mathrm{~s}$} \\
\hline
\end{tabular}

TABLE III.

DETAILS OF TRAINING METHODS

\begin{tabular}{|c|c|c|c|}
\hline Method & $\begin{array}{c}\text { Initial Training } \\
\text { Sessions }\end{array}$ & $\begin{array}{c}\text { Retraining } \\
\text { (Sessions Used) }\end{array}$ & $\begin{array}{c}\text { Total Sessions } \\
\text { Trained }\end{array}$ \\
\hline 1 & 1 to 5 & No & 5 \\
\hline 2 & 1 to 10 & No & 10 \\
\hline 3 & 1 to 3 & Once $(26 \& 27)$ & 5 \\
\hline 4 & 1 to 8 & Once $(26 \& 27)$ & 10 \\
\hline
\end{tabular}

sessions, the system accuracy was evaluated using four different types of training method. The accuracy is shown in Fig. 14. The training methods are distinguished by: a) total number of sessions trained; b) retraining used. Their details are listed in Table III. The preliminary results in Fig. 14 indicate that unintentional gesture variations, which are unavoidable during practical use, can reduce system accuracy. However, this variation could be compensated by using multiple sessions for initial training (occasional retraining also proved to be effective).

The reproducibility of the system was also examined. First, it was investigated whether the accuracy of the results in Section IV-B can be repeated over time. For this test, 6 sessions were carried out by 5 volunteers. In each session, 5 gestures were taken and for each gesture 50 datasets were captured for training and 100 datasets for testing, using group A gestures. Sessions were evenly divided into 3 day intervals with at least 2 hours gap between each session. Using the data from each session for training and testing, the overall accuracy for each user can be plotted as shown in Fig. 15. The results suggest that the system performance of the testing in Section IV-B is reproducible.

Secondly, the correlation between these 6 sessions was also investigated by using the first 5 sessions as training data to test the last session. The results indicate that there is little correlation between the first 5 sessions and the last session. This suggests that initial training is required each time the user starts to wear the device.

Finally, using datasets from the reproducibility test, all data were combined and sent to 12 different machine learning algorithms for performance comparison using the Classification Learner APP in MATLAB in addition to the artificial neural network (ANN) algorithms. With the default setting, each algorithm was trained and the test was done using an exported

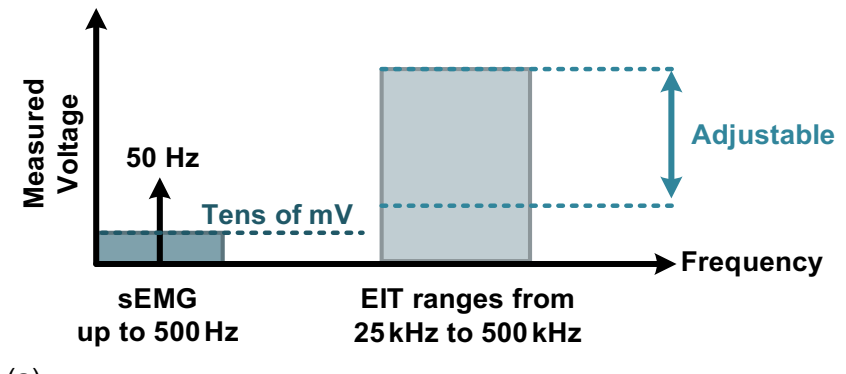

(a)
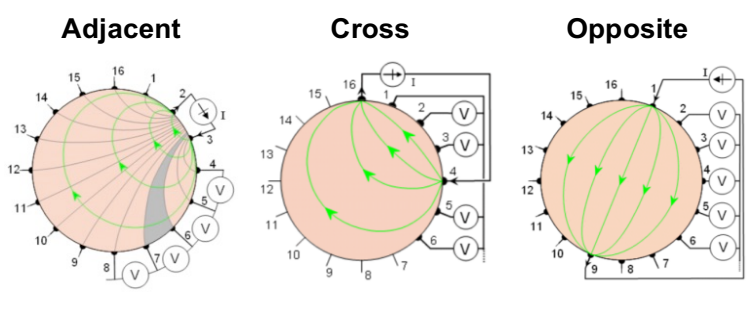

(b)

Fig. 16. Advantages of EIT as an alternative HMI method for hand prosthesis control in terms of: (a) signal bandwidth and strength; (b) different measurement sequences.

model. The results are summarized in Table IV. For each algorithm, the accuracy and computational time are listed. As shown both the support vector machine (SVM) and K-nearest neighbors (KNN) algorithms can reach an accuracy over $90 \%$, but on average KNN tends to require a longer training time. Compared with ANN (with the default setting using 10 hidden neurons) it only takes $1 \mathrm{~s}$ for training while offering competitive accuracy; by increasing the neuron size to 100 its accuracy increased to $91.1 \%$ while requiring less training time compared with other algorithms. These preliminary results suggest that the EIT datasets are not particularly sensitive to any specific machine learning algorithm. However it is possible that optimization of a specific algorithm will offer better accuracy with shorter training times in the future. 


\section{DisCUSSION AND Future Work}

The proposed EIT HMI system differentiates hand gestures using the differences in bio-impedance. For the system to work reliably, it is essential that the impedance features measured from each gesture for training have good generality and remain unchanged from session to session. Based on the preliminary results in Section IV impedance features can vary due to different ways of performing the same gesture. Fortunately, this alteration can be compensated for a single user. The other likely impedance feature alteration can be caused by different electrode wristband positioning. For example, the electrodes 6 and 7 in Fig. 16(b) measure the impedance over the gray shaded area in the first session. In a following session the wristband position will be different and the position of electrodes is changed. The system measures different tissue which may possess very different impedance values. Such alteration has been observed during the second part of the medium-term performance where the user not only took off the band from session to session, but also the electrode position was constantly adjusted to fit different users. As a result, even though the system is able to function properly within sessions, little correlation is found between sessions. This leads to an unavoidable initial training each time the band is worn.

Despite the training only requiring a few minutes, it is still a weakness of this HMI system. Given the limited number of sessions of this preliminary study, further work is needed to examine whether these feature alterations due to electrode band positioning could be improved. Similar issues are faced by the sEMG method where electrode position markers are used when carrying out chronic tests [33]. In addition, to improve the system chronic performances, adaptive algorithms [34] and feedback method [35] are also proposed towards solving this open challenge. These solutions may be considered to be transferrable when using the EIT method.

Compared to sEMG whose amplitude ranges up to tens of $\mathrm{mV}$ with frequencies up to about $500 \mathrm{~Hz}$, as shown in Fig. 16(a), the frequency and the amplitude of measurable voltages in EIT are directly related to the current drive which is user programmable in the range from hundreds of $\mu \mathrm{A}_{\mathrm{p}-\mathrm{p}}$ to a few $\mathrm{mA}_{\mathrm{p}-\mathrm{p}}$ and in a bandwidth from $25 \mathrm{kHz}$ up to $500 \mathrm{kHz}$. This means the amplitude of the induced measurable signal is adjustable in favor of producing a higher signal-to-noise ratio. Also as the frequency of the signal is tunable, it can be moved away from the low-frequency interferences, such as the $50 \mathrm{~Hz}$ mains, which makes the system more robust. In addition, as different types of human tissue, e.g. bone, fat or muscles, feature different values of bio-impedance due to their biological structures, with EIT they can be differentiated at different frequencies [36]. Interrogation of tissues with a wide and tunable bandwidth could offer more information. EIT can also operate with different measurement sequences [37], [38]. It could interrogate different or deep muscle groups with different sequences for more in-depth analysis as shown in Fig. 16(b). Finally, EIT could not only offer high-resolution electrode arrays but also multi-plane measurements (otherwise known as three-dimensional (3D) EIT [39]) which could gather impedance variations at different arm positions simultaneously. Impedance frequency differentiation, exploring measurement sequences and high-resolution multi-plane (3D) EIT hardware upgrades, as well as a performance comparison between sEMG and EIT will be the subject of future investigation.

\section{CONCLUSION}

In this paper, a HMI based on EIT technology has been developed. The EIT reader uses a high-performance ASIC that consists of a fully differential current driver and a low noise, high CMRR IA. The ASIC has a bio-impedance sensitivity of at least $12.7 \mathrm{~m} \Omega_{\mathrm{p}-\mathrm{p}}$. When integrated into the EIT reader, it can accurately capture the user's forearm inner bio-impedance redistribution, providing both real and imaginary impedance readings to the $\mathrm{PC}$ for pattern recognition and feature extraction using an artificial neural network. The system can recognize eleven hand gestures with a gesture enabled sub-grouping method to enlarge gesture pool sizes while ensure recognition accuracy with minimum influence on user experiences. Experiments have shown that the system can achieve $98.5 \%$ accuracy with a grouping of three gestures and an accuracy of $94.4 \%$ with two sets of five gestures. A complete HMI system based on the EIT principle for hand prosthesis control has been presented, and its medium-term performance investigated including a preliminary analysis over different types of machine learning algorithm. The merits as well as the limits of EIT as an HMI system have also been discussed and future work proposed.

\section{REFERENCES}

[1] H. Liu and L. Wang, "Gesture recognition for human-robot collaboration: A review," Int. J. Ind. Ergon., https://doi.org/10.1016/j.ergon.2017.02.004.

[2] S. S. Rautaray and A. Agrawal, "Vision based hand gesture recognition for human computer interaction: a survey," Artif. Intell. Rev., vol. 43, no. 1, pp. 1-54, 2012.

[3] P. Molchanov, S. Gupta, K. Kim, and K. Pulli, "Multi-sensor system for driver's hand-gesture recognition," in Proc. 2015 11th IEEE Int. Conf. and Workshops on Automatic Face and Gesture Recognition (FG 2015), Ljubljana, Slovenia, 2015.

[4] X. Li, R. Wen, Z. Shen, Z. Wang, K. D. K. Luk, and Y. Hu, "A wearable detector for simultaneous finger joint motion measurement," IEEE Trans. Biomed. Circuits Syst., vol. 12, no. 3, pp. 644-654, 2018.

[5] A. Fougner, O. Stavdahl, P. J. Kyberd, Y. G. Losier, and P. A. Parker, "Control of upper limb prostheses: Terminology and proportional myoelectric controla review," IEEE Trans. Neural Syst. Rehabil. Eng., vol. 20, no. 5, pp. 663-677, 2012.

[6] T. Labs, "Myo-An wearable gesture control and motion control device," 2018. [Online]. Available: https://www.myo.com/.

[7] M. Yoshikawa, M. Mikawa, and K. Tanaka, "A myoelectric interface for robotic hand control using support vector machine," in Proc. 2007 IEEE/RSJ Int. Con. on Intelligent Robots and Systems, San Diego, CA, pp. 2723-2728.

[8] G. Tsenov, A. H. Zeghbib, F. Palis, N. Shoylev, and V. Mladenov, "Neural networks for online classification of hand and finger movements using surface EMG signals," in Proc. 8th Seminar on Neural Network Applications in Electrical Engineering (NEUREL 2006), Belgrade, Serbia and Montenegro, Serbia, 2006, pp. 167-171.

[9] G. Huang, Z. Zhang, D. Zhang, and X. Zhu, "Spatio-spectral filters for low-density surface electromyographic signal classification," Med. Biol. Eng. Comput., vol. 51, no. 5, pp. 547-555, 2013.

[10] C. Cipriani et al., "Online myoelectric control of a dexterous hand prosthesis by transradial amputees," IEEE Trans. Neural Syst. Rehabil. Eng., vol. 19, no. 3, pp. 260-270, 2011.

[11] P. Geethanjali and K. K. Ray, "A low-cost real-time research platform for EMG pattern recognition-based prosthetic hand," IEEE/ASME Trans. Mechatronics, vol. 20, no. 4, pp. 1948-1955, 2015.

[12] S. L. Teng, R. Rieger, and Y. Bin Lin, "Programmable ExG biopotential front-end IC for wearable applications," IEEE Trans. Biomed. Circuits Syst., vol. 8, no. 4, pp. 543-551, 2014. 
[13] S. Benatti et al., "A versatile embedded platform for EMG acquisition and gesture recognition," IEEE Trans. Biomed. Circuits Syst., vol. 9, no. 5, pp. 620-630, 2015 .

[14] A. Andrews, E. Morin, and L. McLean, "Optimal electrode configurations for finger movement classification using EMG," in Proc. 2009 IEEE Eng Med Biol Conf. (EMBC 2009), Minneapolis, MN, 2009, pp. 2987-2990.

[15] G. Li, Y. Geng, D. Tao, and P. Zhou, "Performance of electromyography recorded using textile electrodes in classifying arm movements," in Proc 2011 IEEE Eng Med Biol Conf. (EMBC 2011), Boston, MA, 2011, pp. $4243-4246$.

[16] M. a. Oskoei and H. H. H. Hu, "Support vector machine-based classification scheme for myoelectric control applied to upper limb," IEEE Trans. Biomed. Eng., vol. 55, no. 8, pp. 1956-1965, 2008.

[17] M. Atzori, M. Cognolato, and H. Müller, "Deep learning with convolutional neural networks applied to electromyography data: A resource for the classification of movements for prosthetic hands," Front. Neurorobot., vol. 10, no. 9, 2016.

[18] A. M. Simon, K. Stern, and L. J. Hargrove, "A comparison of proportional control methods for pattern recognition control," in Proc 2011 IEEE Eng Med Biol Conf. (EMBC 2011), Boston, MA, 2011, pp. 3354-3357.

[19] C. Castellini and P. Van Der Smagt, "Surface EMG in advanced hand prosthetics," Biol. Cybern., vol. 100, no. 1, pp. 35-47, 2009.

[20] P. F. Pasquina et al., "First-in-man demonstration of a fully implanted myoelectric sensors system to control an advanced electromechanical prosthetic hand," J. Neurosci. Methods, vol. 244, pp. 85-93, 2015.

[21] H. Gagnon, M. Cousineau, A. Adler, and A. E. Hartinger, "A resistive mesh phantom for assessing the performance of EIT systems," IEEE Trans. Biomed. Eng., vol. 57, no. 9, pp. 2257-2266, 2010.

[22] P. J. Langlois, Y. Wu, R. H. Bayford, and A. Demosthenous, "On the application of frequency selective common mode feedback for multifrequency EIT," Physiol. Meas., 2015, vol. 36, no. 6, pp. 13371350.

[23] J. Rosell and P. Riu, "Common-mode feedback in electrical impedance tomography," Clin. Phys. Physiol. Meas., vol. 13, pp. 11-14, 1992.

[24] Y. Zhang and C. Harrison, "Tomo: Wearable, low-cost, electrical impedance tomography for hand gesture recognition," in Proc. 28th Annual ACM Symp. User Interface Softw. Technol. (UIST 2015), Charlotte, NC, 2015, pp. 167-173.

[25] Y. Zhang, R. Xiao, and C. Harrison, "Advancing hand gesture recognition with high resolution electrical impedance tomography," in Proc. 29th Annual Symp. on User Interface Software and Technology (UIST 2016), Tokyo, Japan, 2016, pp. 843-850.

[26] Y. Wu, D. Jiang, J. Duan, X. Liu, R. Bayford, and A. Demosthenous, "Towards a high accuracy wearable hand gesture recognition system using EIT," in Proc. 2018 IEEE Int. Symp. Circuits Syst. (ISCAS 2018), Florence, Italy, 2018.
[27] H. Hong, M. Rahal, A. Demosthenous, and R. H. Bayford, "Comparison of a new integrated current source with the modified howland circuit for EIT applications," Physiol. Meas., vol. 30, no. 10, 2009.

[28] L. Constantinou, I. F. Triantis, R. Bayford, and A. Demosthenous, "High-power CMOS current driver with accurate transconductance for electrical impedance tomography," IEEE Trans. Biomed. Circuits Syst., vol. 8, no. 4, pp. 575-583, 2014.

[29] Y. Wu, D. Jiang, P. Langlois, R. Bayford, and A. Demosthenous, "A CMOS current driver with built-in common-mode signal reduction capability for EIT," in Proc. ESSCIRC 2017, Leuven, Belgium, 2017, pp. 227-230.

[30] A. Worapishet, A. Demosthenous, and X. Liu, "A CMOS instrumentation amplifier with 90-dB CMRR at 2-MHz using capacitive neutralization: Analysis, design considerations, and implementation," IEEE Trans. Circuits Syst. I Regular Papers, vol. 58, no. 4, pp. 699-710, 2011.

[31] R. D. Cook, G. J. Saulnier, D. G. Gisser, J. C. Goble, J. C. Newell, and D. Isaacson, "ACT3: A high-speed, high-precision electrical impedance tomograph," IEEE Trans. Biomed. Eng., vol. 41, no. 8, pp. 713-722, 1994.

[32] P. Kassanos, I. F. Triantis, and A. Demosthenous, "A CMOS magnitude/phase measurement chip for impedance spectroscopy," IEEE Sens. J., vol. 13, no. 6, pp. 2229-2236, 2013.

[33] J. He, D. Zhang, N. Jiang, X. Sheng, D. Farina, and X. Zhu, "User adaptation in long-term, open-loop myoelectric training: Implications for EMG pattern recognition in prosthesis control," J. Neural Eng., vol. 12, no. $4,2015$.

[34] J. W. Sensinger, B. A. Lock, and T. A. Kuiken, "Adaptive pattern recognition of myoelectric signals: Exploration of conceptual framework and practical algorithms," IEEE Trans. Neural Syst. Rehabil. Eng., vol. 17 , no. 3, pp. 270-278, 2009.

[35] M. A. Powell, R. R. Kaliki, and N. V. Thakor, "User training for pattern recognition-based myoelectric prostheses: Improving phantom limb movement consistency and distinguishability," IEEE Trans. Neural Syst. Rehabil. Eng., vol. 23, no. 3, pp. 522-532, 2014.

[36] T. J. C. Faes, H. A. Van Der Meij, J. C. De Munck, and R. M. Heethaar, "The electric resistivity of human tissues $(100 \mathrm{~Hz}-10 \mathrm{MHz})$ : A metaanalysis of review studies," Physiol. Meas., vol. 20, no. 4. 1999.

[37] J. Malmivuo and R. Plonsey, Bioelectromagnetism - Principles and Applications of Bioelectric and Biomagnetis Fields, Chapter: 26. Oxford University Press, 1995.

[38] S. Russo, S. Nefti-Meziani, N. Carbonaro, and A. Tognetti, "A quantitative evaluation of drive pattern selection for optimizing EITbased stretchable sensors," Sensors (Switzerland), vol. 17, no. 9, 2017.

[39] B. Grychtol, B. Müller, and A. Adler, "3D EIT image reconstruction with GREIT,” Physiol. Meas., vol. 37, no. 6, pp. 785-800, 2016. 